\title{
La concepción de sujeto en la trama de la política social
}

\section{The concept of subject in the frame of social policy}

Claudia Sandra Krmpotic ${ }^{1}$

\section{RESUMEN}

Se analiza la concepción de sujeto que subyace en la definición de las políticas, a fin de repensar la política social desde otro ángulo. La política social tradicional así como otras alternativas más progresistas han definido sus objetivos en función de un sujeto concebido como 'racional' y 'autónomo'. Así las cosas, se procura profundizar en estas características, colocando en foco al sujeto y sus necesidades en clave 'emocional'. A partir de una problematización teórica del 'otro en estado de necesidad', se pondrán en consideración aproximaciones conceptuales y prácticas al problema de la racionalidad y la autonomía, así como aspectos emocionales ligados al relacionamiento, el lenguaje, el sufrimiento, la ambivalencia y la dependencia, en una perspectiva humanista, crítica e interdisciplinaria.

Palabras-clave: Sujeto. Política social. Autonomía. Dependencia. Sufrimiento social.

\section{ABSTRACT}

We analyze the conception of the subject that underlies the definition of policies in order to rethink social policy from another angle. Traditional social policy as well as other progressive alternatives have defined their objectives in terms of a subject conceived as 'rational' and 'autonomous'. So, it seeks to deepen these features, putting in focus the subject and needs in 'emotional' key. From a theoretical problematization of the 'other in need', it will consider the conceptual and practical approaches to the problem of rationality and autonomy, and emotional issues related to relationships, language, suffering, ambivalence and dependence in a humanistic, critical and interdisciplinary perspective.

Keywords: Subject. Social policy. Autonomy. Dependence. Social suffering.

\footnotetext{
${ }^{1}$ Investigador CONICET, Centro Argentino de Etnología Americana/Consejo Nacional de Investigaciones Científicas y técnicas), Prof. Universidades Nacionales de Buenos Aires y La Matanza. Postdoctorado en Cs. Humanas (UFSC-BR); Doctorado en Servicio Social (PUC,SP-BR); Magister en Ciencia Política (Fundación Banco Patricios-AR); Licenciada en Servicio Social (UBA-AR)
}

Esta obra foi licenciada com uma Licença Creative Commons - Atribuição 3.0 Não Adaptada. 


\title{
1 INTRODUCCIÓN
}

Con la decadencia de los mecanismos garantes del orden social aparecen los cuestionamientos, y el desencantamiento del mundo -según la expresión de Weberse reaviva. Con esta descripción introduce Touraine (1987) su título El regreso del actor, y explica de la siguiente manera la tarea por delante:

\begin{abstract}
El regreso del sujeto como quizás este libro hubiera debido llamarse... y continúa... lo esencial es sin duda la necesidad de definir nuevamente al sujeto, no tanto ya por su capacidad de dominar y transformar el mundo, sino por la distancia que toma en relación con esa capacidad y con los aparatos y discursos que la establecen (TOURAINE, 1987, p. 19).
\end{abstract}

Estas ideas escritas en el Pentecostés de 1984, encontrarán eco en toda la literatura posterior acerca del proceso de individualización de lo social, conservando algunas preocupaciones en común. Con la crítica al individuo de la llustración, posesivo y egotista, que 'es individuo' en una lógica de estructuras, frente a aquel 'que se hace individuo' en la segunda modernidad en base a una lógica de flujos, Lash enfatiza aspectos de la obra de Beck y Beck-Gernsheim (2003) explicando cómo el sujeto liberal se inscribe en una sociedad en tanto sistema lineal que tras perturbaciones externas, cambia y se adapta, mientras que la actual individualización implica una desestabilización del sistema social. Uno y otro sujeto es analizado en el contraste entre reflejo y reflexión, lo que conduce a pensar al sujeto post-liberal como un sujeto en movimiento en base a un conocimiento de incertidumbre; volviendo a Lash (2003, p. 13)

\footnotetext{
lo que tenemos ahora no es un no-conocimiento o una anti-razón... lo que ha cambiado es el tipo de conocimiento en cuestión. Es de por sí precario en cuanto que es distinto del conocimiento cierto, y el objeto de dicho conocimiento es también incierto; en el mejor de los casos probabilístico, por no decir más bien posibilístico.
}

Con este espíritu, advertimos que hasta el momento, reflexiones de este orden han influido sólo tangencialmente el debate sobre políticas públicas, con algunos abordajes innovadores desde subdisciplinas como la psicología o la antropología política, y aproximaciones de carácter fenomenológico en general, donde el foco de atención está puesto en el encuentro entre usuarios, ciudadanos y las instituciones y los servicios. Generalmente cuando se analizan los éxitos y fracasos de la Política Social dirigida a grupos vulnerables o con necesidades Cad. de Pesq. Interdisc. em Ci-s. Hum-s., Florianópolis, v.12, n.101, p.31-47 ago/dez 2011 
especiales, se pone el acento en las fortalezas o debilidades en el diseño del programa social, en su concepción del problema, en la selectividad o universalidad, en la cantidad y calidad de los recursos y servicios, en el costo de oportunidad de una evaluación ex -ante y concurrente para ir monitoreando las acciones; en fin, todo un arsenal técnico válido para dar cuenta del proceso de cambio en la vida de personas y grupos sociales, y sus resultados en el mundo real. Al respecto, es propósito de este texto circunscribirnos en cambio, a las expectativas en torno al sujeto destinatario, a la concepción de 'sujeto' que subyace en la definición de las políticas, a fin de repensar la política social desde otro ángulo.

En este sentido, la política social tradicional así como otras alternativas más progresistas han definido sus objetivos en función de un sujeto concebido como 'racional' y 'autónomo'. Así las cosas, se procura profundizar en estas características, colocando en foco al sujeto y sus necesidades en clave 'emocional'. A partir de una problematización teórica del 'otro en estado de necesidad', se pondrán en consideración aproximaciones conceptuales y prácticas al problema de la racionalidad y la autonomía, así como aspectos emocionales ligados al relacionamiento, el lenguaje, el sufrimiento, la ambivalencia y la dependencia, en una perspectiva humanista, crítica e interdisciplinaria.

Si bien se trata de un ejercicio analítico, se retoman observaciones del trabajo en campo con mujeres y hombres adultos y jóvenes, de barrios periféricos del Gran Buenos Aires, como fuente para sugerir los interrogantes que ordenan este material. Sus vidas cotidianas reflejan en sus múltiples aristas y heterogéneas prácticas, discriminación, falta de oportunidades, sufrimiento, muerte joven, ausencia de trabajo regular por ya casi tres generaciones en grupos familiares jóvenes, como una presencia de la espiritualidad y apelaciones a la creencia religiosa en sentido amplio.

\section{UN SUJETO RACIONAL Y AUTÓNOMO}

A propósito del sujeto producto de la modernidad, señalaremos apenas algunas notas distintivas que nos permitan avanzar en la argumentación. Acompañando la vocación disruptiva de la modernidad, la idea de sujeto expresa 
esa tendencia a la distinción, a la creciente separación entre racionalización y subjetivación, entre lo positivo concreto y lo espiritual, entre el universo de la instrumentalidad y el de la identidad, entre el actor y el sistema. Asimismo, se trata de un sujeto que construye su identidad (que ya no está dada por designios sobrenaturales) resolviendo las tensiones entre los imperativos biológicos (naturaleza) y los imperativos sociales (cultura); se aspira a un hombre que mediante el control de la pasiones y la transformación de aquellas destructoras en virtudes. Como sagazmente describe Hirschman (1978) no se trata de su represión sino -insistimos- de su transformación en algo positivo, en fuerza creadora a favor del bienestar general a partir del interés individual. La 'razón observante' de Hegel, el concepto freudiano de 'sublimación', la 'mano invisible' de Smith ejemplifican la idea de un sujeto que autocontrola su conducta guiado por la racionalidad (o sea, la adecuación medios-fines). Si bien la liberación de las coacciones y formas represivas previas no es total, ya que se recrean mecanismos institucionales de regulación social -como la familia, el trabajo y el Estado- lo cierto es que se asume una nueva subjetividad en base a una moral interiorizada. Ello supone una responsabilidad frente a las contingencias y capacidad de dominio (conciencia) sobre el destino.

De ese sujeto ahora libre y responsable se esperan conductas estables, predecibles y regulares, así como una actitud cooperativa sin la necesidad de coacción permanente o de al menos un mínimo de control. De allí que las modernas políticas sociales propenderán a moldear lo que Lukács (2007) denominara disposiciones secundarias, con referencia a fuerzas psíquicas y sentimientos indispensables para acompañar la nueva división social del trabajo, tanto en el orden privado (sexualidad/personalidad; consumo/cultura), como en el orden público (empresa/economía; estado/política).

La burocratización y profesionalización del Estado, a medida que aumenta su margen de intervención, así como las demandas calificadas del mundo del trabajo, conformaron unas instituciones especializadas las que -a través de las profesiones y la gubernamentalidad- acompañaron en la tarea de moldear un sujeto racional y autónomo.

De un usuario así concebido se esperan unas manifestaciones relativamente ordenadas y unificadas, una identidad consistente y coherente, una actuación 
racional que integre la responsabilidad individual mas también su rol de agencia en la preocupación por terceros y el entorno. Como contrapartida, la carencia de tales atributos nos enfrenta a personas que identificaremos como dependientes, impredecibles, incapaces de actuar en su mejor interés o en beneficio de quienes le rodean, pensaremos en dificultades a la hora de internalizar las normas sociales, etc.

La libertad disponible (en tanto hombres libres) nos obligará a tomar las opciones que -bajo condiciones de autonomía- decidiremos como las más convenientes. Por su parte, tanto la igualdad de oportunidades como la igualdad de resultados, pivotean sobre la información, o más bien, sobre las condiciones para una elección informada. Las políticas entonces no sólo se resumen en la distribución de bienes o servicios, sino particularmente de información, para que la mejor decisión pueda ser alcanzada por el individuo. Incluso, todo un vasto desarrollo en torno de las amenazas a la autonomía se verá reflejado en conceptos y explicaciones que intentan resolver este cuello de botella clave para la política social: las filtraciones, los costos burocráticos, el clientelismo y la corrupción, como el aumento de la racionalidad y la lucha contra la dependencia de los pobres de los programas sociales, serán búsquedas constantes.

Si nuestra hipótesis -al reubicar el problema en la noción de sujeto- encuentra asidero, podemos en consecuencia poner en duda la eficacia simbólica de dichas intervenciones, y en general, el poder del aparato estatal. La ilusión ingenieril puede estar 'jugándonos una mala pasada' al hacernos creer en que efectivamente la acción de las políticas modifica la vida de las personas según lo planeado. La mirada estratégica de las últimas décadas trató de superar esa ilusión, al incorporar un rol activo de ese usuario ahora convertido en agente, que actúa estratégicamente en función de su conveniencia y la de su grupo. No obstante, ya Heller y Ferenc (1995) señalaban que la familia no se somete fácilmente; esta resiste y constituye el vestigio más poderoso de la naturaleza en el enclave de lo social: sus prácticas siempre tienden a transgredir, nos demuestran la fuerza de los factores naturales y su socialización (edad, sexo, capacidades, talentos, etc.) y el propio objetivo no siempre alcanzado de 'desnaturalizar' de esquemas de pensamiento y comportamiento nos advierte, como contrapartida, la presencia de aspectos que por momentos parecen pulsiones que juegan de modo determinante. Desde otra 
perspectiva, DiLiscia y Bohoslavsky (2005) también sugirieron -con validez para experiencias locales- una valoración desmedida de las capacidades materiales y simbólicas del Estado y grupos dominantes, al recuperar la presión y los saberes de los grupos subordinados para influir, resistir o resignificar la agenda implementada desde arriba, y las consecuentes posibilidades de negociación. Hay dominación pero también interferencias, resistencias y transformación. El sentido del propio concepto de 'prácticas' (políticas, sociales, institucionales) permite resaltar esa oscilación entre su carácter tanto naturalizador como crítico.

\section{UN SUJETO EMOCIONAL}

Ahora veamos el problema desde una perspectiva diferente colocando en foco al sujeto y su vida 'emocional'. Frost y Hoggett (2008) invitan a pensar en ese sentido en un sujeto post-liberal. Si consideramos al sujeto con capacidad de 'relacionamiento' en lugar de autonomía, ello nos obliga a revisar la categoría de dependencia en la medida que en cualquier condición precisamos del 'otro', en nuestra precaria e incompleta unidad del existir. En este caso, la cuestión de la 'otredad' sintetiza el nudo gordiano de la asistencia social: el 'otro' genera tanto hospitalidad como hostilidad ${ }^{2}$ : por un lado lo tratamos como un par, que logre sentirse como 'en su casa', y por momentos emerge el carácter de extraño, de ajeno, o sea, de posible amenaza hostil. Entre la confianza y el temor oscilan las actitudes presentes tanto en los intercambios simples, como en aquellas acciones políticas nacionales a través de sus gestores, de los medios de comunicación, de los contribuyentes, etc. Incluso en esta relación inevitable se dirimen las diferencias sociales, por cuanto quien hospeda presenta una posición, un 'aquí', una morada, que el otro requiere.

En esta perspectiva, la razón junto a las pasiones se encarnan o corporizan e integran (en lugar de considerarse opuestas) en un sujeto que no siempre se maneja con racionalidad; se reconoce a un ser 'ambivalente', conducido 'emocionalmente',

\footnotetext{
${ }^{2}$ Recordemos que el término 'hostis' se puede traducir al español como huésped o como hostil y enemigo.

Cad. de Pesq. Interdisc. em Ci-s. Hum-s., Florianópolis, v.12, n.101, p.31-47 ago/dez 2011
} 
que existe más allá -pero definido- en el marco del 'lenguaje'. La experiencia subjetiva como intersubjetiva emergen como relevantes para explicar la objetivación en actos y materiales de la cultura; todos resultados no sólo del pensar (racional), sino además del sentir, del responder, y el actuar.

Por su parte, si aceptamos que la satisfacción de necesidades transcurre en la vida cotidiana, ello nos define una escala en la que resulta difícil transpolar las pretensiones de racionalidad y autonomía. Entendida como 'dureé', la cotidianeidad es un fluir de vivencias; metafóricamente, una suerte de río en el que estamos embarcados más o menos reflexivamente, llevados por las percepciones, esquemas de comportamiento y de pensamiento derivados de nuestras experiencias. En ese transcurrir en tiempo presente se registran eventos de baja excepcionalidad (de allí esos rasgos de repetición, imitación y naturalidad que diversos autores coinciden en señalar), de movimientos lentos, con momentos de ruptura o dislocación, que rápidamente serán contrarrestados por mecanismos de estabilización. Bergson (1900) ha definido las vivencias cotidianas oscilantes entre dos actitudes: una atención a la vida cuando en uso de la conciencia espacio-temporal ello nos impide sumergirnos en la 'dureé'; y otra, cuando se relaja la tensión psíquica, dejándonos llevar por el fluir indiferenciado y continuo de las vivencias. El acto reflexivo altera la corriente de conciencia, y es sólo entonces cuando el flujo de sucesos se convierte en objeto de reflexión, es decir, adquiere entidad discreta y significado, y puede transformar en un sentido estabilizador como crítico. Los estudios sobre vida cotidiana, como los enfoques fenomenológicos y constructivistas, aportan argumentos suficientes para identificar unos límites claros a la racionalidad impuesta desde la concepción de sujeto liberal que procuramos problematizar.

Entonces, ¿que implica intervenir en las experiencias de este otro tipo de sujeto? En un sentido fenomenológico puede decirse que las experiencias sintetizan tres dimensiones de la vida: una material, una actitudinal y una simbólica e imaginaria, de modo que cualquier intervención afecta a los tres aspectos. El tipo de experiencias conducirá por momentos -en ese fluir de la cotidianeidad- al razonamiento acerca de las condiciones en que la vida de cada uno se inscribe. A modo de ejemplo, no es sólo la falta de ciertos alimentos en la mesa familiar lo que define las carencias en un hogar, sino el razonamiento sobre ello, lo que constituye una práctica concreta, no apenas un significado o representación anexa: esa es la 
experiencia de malestar. Por tanto, si cambiamos el punto de partida, las carencias o déficits en la conformación de la identidad y el aparato psíquico, que en términos individuales inhabilitan a los sujetos a valerse por sus propios medios y garantizar con su trabajo la satisfacción de necesidades según la concepción anterior, serán reemplazadas por una valoración del 'malestar físico y psíquico' y del 'sufrimiento social' que provocan. Ya Doyal y Gough (1994) habían reparado hace un tiempo en que la posesión de satisfactores materiales no explicaban las necesidades humanas fundamentales. Los primeros fueron definidos como necesidades intermedias, mientras que la supervivencia o salud física, y la autonomía o salud mental, consideradas las necesidades fundamentales por cuanto definen condiciones previas básicas para evitar prejuicios graves o el aumento del deterioro en una forma de vida, todo lo que impide la práctica de una participación sostenida. Ahora veamos el aspecto de la autonomía, que viene siendo puesto en tela de juicio. Visto desde el desempeño del sujeto (en lugar de las políticas), los autores la definen como la capacidad de formular objetivos y estrategias consistentes, y a los intentos de ponerlas en práctica en las actividades que se emprenden. Incluye dimensiones como el grado de comprensión que la persona tiene de sí misma, de su cultura y de lo que se espera de ella dentro de la misma; la capacidad psicológica para formular opciones; y las oportunidades objetivas que les permitan actuar en consecuencia. De la autonomía se desprenden las razones por las cuales consideramos a una persona práctica y moralmente responsable de sus acciones. Reaparece la racionalidad, lo cual nos retrotrae al punto de partida. Variantes relativistas discutirán el significado de salud física y mental, en el intento por desmantelar la creencia en las regularidades nomológicas como aspecto del sujeto liberal secular, universalizable a través del entendimiento técnico y del análisis causal. Estas aproximaciones pondrían en jaque la noción de elección racional pues se hace imposible si existen diferentes criterios como subproducto de las diversas formas de comprensión de un malestar o una enfermedad. 


\section{EL SUFRIMIENTO COMO CATEGORÍA DESESTABILIZADORA}

Propongo detenernos en un concepto que pone de manifiesto la intersección de disciplinas y miradas. En las ciencias sociales, ha sido Bourdieu (1999) uno de los que llamó la atención sobre el concepto de sufrimiento social como camino para alcanzar un análisis psicosocial del objeto del bienestar. No sólo entendido como resultado de la desigual distribución de bienes (tal como la política bienestarista ha tendido a enfatizar), sino además de ello, a partir de las vivencias de dominación y represión. Se incluyen sentimientos de humillación, ira, resentimiento, desesperanza, que acompañan las manifestaciones de la cuestión social. Sufrimiento en tanto fenómeno reflexivo como no-reflexivo: como algo que por momentos puede ser pensado crítica y creativamente, mientras que en ocasiones se materializa y proyecta como algo sobre lo que apenas se puede razonar y menos justificar. Cuando enfatizamos su carácter estructural hacemos hincapié en un fenómeno multicausal, crónico, que puede ir desde el hambre, las enfermedades, la violencia y la tortura, hasta la muerte, que restringe y hasta clausura las elecciones de vida, originado al menos en tres dimensiones preponderantes en el marco de una historia regional: el eje de género, el de la etnicidad, y el del status socio-económico (FARMER, 1997).

El sufrimiento constituye una categoría desestabilizadora, generalmente no considerada en el estudio de la dialéctica exclusión/inclusión, pero relevante pues nos permite cualificar, dotar de intensidad a las vivencias que objetivamente -en la distancia científica y profesional- intentamos describir en los diagnósticos sociales (SAWAIA, 2001). Para muchos 'cada día se siente como en la guerra', o 'puede ser el último', de allí que Merklen (2005) retome la metáfora del 'cazador' -ahora urbanopara dar cuenta no de un indigente pasivo, sino de un agente activo, especializado y conocedor, que encuentra en su hábitat popular, en el barrio, a través de la multiactividad, los recursos para la autosubsistencia en el día a día. Lógica de individualización pero inscripta en una acción en su comunidad de pertenencia ${ }^{3}$.

\footnotetext{
${ }^{3}$ La metáfora del cazador según el autor “... permite identificar una figura de individuo y una lógica de acción, y la caza designa una situación de individuación”; asimismo "en primer lugar donde reina la precariedad, el cazador traduce una forma adaptativa en condiciones de vida extremadamente Cad. de Pesq. Interdisc. em Ci-s. Hum-s., Florianópolis, v.12, n.101, p.31-47 ago/dez 2011
} 
La idea de sufrimiento social incluye problemas que son resultado de daños infligidos por las fuerzas sociales sobre la experiencia humana. Implica pensar en lo que el poder político, económico e institucional hace a las personas, $y$ recíprocamente, cómo esas formas de poder influencian el modo de responder a tales problemas, a los que en general tratamos como campos separados, en términos de salud, bienestar, problemas legales, morales o religiosos. A su vez, nos permite ver al otro en su 'humanidad', lo que implica colocar al sujeto en el centro de un análisis que incluye relaciones de poder, cierre de oportunidades, privación económica, más también, deseos, expectativas, tiempo vital y afectividad.

Sus efectos pueden ser descriptos como 'daños' (heridas ocultas de la clase, el género, el origen étnico; el estigma y la falta de reconocimiento; el daño internalizado) y como 'pérdidas' (materiales, del empleo, de la estabilidad económica, las migraciones forzosas; de las pérdidas físicas; pérdidas de humanidad en aquellas poblaciones subalternizadas entre quienes son elementos constitutivos de la identidad; del sufrimiento corporizado y expresado en la sintomatología física y la somatización). A pesar de no darnos cuenta, las emociones resultan en potentes ideologemas que muchas veces tienen la función de mantener un orden social excluyente, tal el caso de la vergüenza y la culpa. Nos preguntamos ¿cómo poder trabajar con alguien si el punto de partida es su sentimiento de culpa o la vergüenza frente a determinada situación?, ¿en qué medida esperar su compromiso con la visión del cambio, de qué manera pautar una relación profesional lo menos asimétrica posible?

Es difícil abordar la cuestión del sufrimiento: primero, por la distancia con aquellos que han sufrido por varias generaciones, y que hace a una menor capacidad de comprensión del observador; segundo, el sufrimiento no siempre se puede poner en palabras o en figuras que lo objetiven, sus víctimas con anónimas y sus voces ni escuchadas; y tercero, apenas se ha alcanzado a explicar el sufrimiento del enfermo; el estudio de tales vivencias sólo puede realizarse a través de una inmersión en la biografía personal sin perder de vista los determinantes de la matriz cultural, histórica y política en que el sufriente ha vivido.

inestables; en segundo lugar la figura del cazador responde a formas de inscripción colectiva que permiten a estas personas existir, mal o bien, como individuos" (MERKLEN, 2005, p.176)

Cad. de Pesq. Interdisc. em Ci-s. Hum-s., Florianópolis, v.12, n.101, p.31-47 ago/dez 2011 


\section{CONSECUENCIAS PRÁCTICAS PARA LA ACCIÓN}

Como señalé en otro lugar, si efectivamente enfrentamos una nueva etapa, "las tres dimensiones del cambio epocal se verán afectadas: el campo de la experiencia, el de las estructuras sociales y el de las representaciones culturales" (KRMPOTIC, 2008, p. 32), de modo que en esas intersecciones deben resignificarse los alcances de la política social.

Ante un sujeto básico racional y autónomo la política se restringe, en la medida que el bienestar es entendido como un óptimo en la producción y distribución de bienes materiales y servicios que permitan ampliar las opciones de vida de los individuos, y sus fines son definidos en términos del manejo del sufrimiento ante riesgos previsibles y calculables. Sin embargo, cuando la capacidad de dominio sobre los factores que nos ponen en riesgo se acerca o es igual a cero, tales condiciones dejan de jugar como 'riesgo de', y pasan a ser sufrimiento. Y si esas condiciones se hacen crónicas, cotidianas, continuas, inherentes al 'modo de vida', no hay contingencia, no lo puedo evitar: estamos allí ante la necesidad de alivio del sufrimiento.

Asimismo se universalizan los medios e instrumentos dirigidos a un individuo 'tipo'. Por su parte, la noción de autonomía refiere básicamente a la condición de aquel cuanto más libremente pueda llevar adelante sus decisiones sin estar sometido al imperio de la necesidad. La politización de las necesidades, y la estatización de las respuestas también serán valoradas según criterios de racionalidad a fin de justificar la ayuda a los dependientes, más allá de la simple o mera cuestión de humanidad. Los que dan -sean los ricos, los que pagan impuestos, los votantes- vuelven una y otra vez sobre la misma pregunta: ¿es que los pobres solo se aprovechan del trabajo de los demás? (lógica económica, de ganadores y perdedores), ¿se lo merecen? (la ayuda como premio: lógica filantrópica y de las primeras formas de la asistencia social), ¿tienen derechos? (institucionalización de las demandas sociales e influencia creciente de los organismos internacionales bajo la promesa de una paz global). Como sea, estos argumentos no se fundan en las opiniones de los sujetos, sino en las mediaciones jurídico-políticas que dan forma a 
los arreglos institucionales. El sujeto es verdaderamente secundario, es el asistido, el que recibe, ahora con un rol más activo a partir de las categorías de consumidor y ciudadano.

Estos aspectos entre otros han sido problematizados -felizmente- en los últimos 30 años, planteando controversias que congregaron a un gran número de investigadores, a saber: a) la relevancia de comprender transcursos históricos distintos y dimensiones político-ideológicas para identificar modelos de política social en los espacios domésticos más allá de las semejanzas globales en los bienes y servicios colocados al acceso de la población por los Estados de bienestar; b) los límites del proceso de cuantificación de las necesidades con la apertura 'seniana' de fuerte impacto con el concepto de capacidades, además de una revisión de la idea de justicia distributiva a partir de otras variantes, como en el caso de la discriminación positiva; c) el cuestionamiento a la lógica de distribución de personas, posiciones y riqueza, en tanto background del desarrollo moderno; d) el reconocimiento de los vínculos entre lazo social y monetario, recuperando reflexiones presentes en la alborada de los mercados en la relación entre caridad y economía, entre don y contrato, advirtiendo acerca de la degradación de la sociedad en la medida que se extienda la lógica del mercado a todas las áreas de intercambio; e) la generación post-reforma de políticas sociales (a partir de los noventa) con énfasis en los activos sociales, el capital humano y social al servicio del desarrollo; y f) el debate acerca de cómo compatibilizar sistemas solidarios basados en derechos y no en tutelas, analizando los efectos de la compasión que hiere, como del respeto y la distancia burocrática, entre otras líneas de investigación.

Reintroduzcamos ahora la cuestión de las 'dependencias básicas' que en esta perspectiva, se identifican en la sociedad moderna respecto del mercado, de la familia y del poder. Ahora bien, ¿crea la sociedad capitalista condiciones para esa realización de la autonomía?. ¿podemos efectivamente ser libres frente a tales espacios de dependencia? ¿es deseable superar la dependencia, o es parte de la condición humana? La paradoja de la cuestión social se presenta con todos sus matices, poniendo en evidencia la tensión inmanente e irresuelta en este tipo de sociedad entre emancipación y regulación.

Volvamos sobre el sentido de la libertad. Libremente significa que -de manera material y simbólica- el sujeto domina sus acciones, en lugar de que las mismas 
sean resultado de condiciones que no controla (DANANI, 2009). Como bien señala la autora, en política social no se trata de cualquier sometimiento sino en particular, del sometimiento a las necesidades de reproducción. De alguna manera se trata de la creencia en el control de las condiciones que nos toca vivir que subyace a la meta de la 'desmercantilización' cuando se intenta independizar la satisfacción de los ingresos o de la capacidad de pago; la 'desfamiliarización', cuando se procura que la capacidad de los individuos para seguir su propia vida se libere de las ataduras familiares, y en el caso de las redes clientelares cuando la finalidad tiene que ver con su 'desarticulación'. Desviaciones en esos tres campos de problemas derivados del diseño como de la gestión, calan hondo en el profuso debate en el campo de la política social frente a usuarios que pueden ver enajenadas tanto su voluntad como su capacidad de agencia.

Como nos propusimos reemplazar la preocupación por la dependencia por el valor del relacionamiento, entonces en lugar de ver el problema de la satisfacción de necesidades como una consecuencia de la distribución del ingreso, podemos pensar en cambio que vivimos en una sociedad que distribuye 'modos o estilos de vida' (BREILH, 2003) según patrones de 'desigualdad' (impedimentos en el acceso a titularidades y recursos) y de 'diversidad' de atributos humanos (naturales y sociales, atinentes al genotipo y al fenotipo). De aceptar esta proposición, el objetivo sería analizar las distintas formas de relacionamiento presentes en los modos o estilos de vida, que conducen al bienestar/malestar, estructuran tales experiencias y explican los sentimientos de dominación y exclusión. En este caso, más que el manejo de sus efectos, la acción planificada debiera pensar en un alivio ante los daños y las pérdidas y en una ampliación de opciones por modos de vida más saludables. Si incorporamos estas variantes, otro será el marco para explicar los fines de la política social respecto de las tres dependencias básicas antes señaladas. En todo caso implicará analizar esos procesos a la luz de los atravesamientos que derivan del estilo de vida, lo que permitirá explicar distintas trayectorias y resultados frente a aquellas intencionalidades políticas.

El modo de organizar el quehacer cotidiano, de alimentarse, de amar, recrearse, de vivir como de enfermar, de buscar el placer como de manejar el dolor, si bien son aspectos que la mayoría han sido regulados por las instituciones, no dejan de interpelar a la cultura y a los sistemas de creencias. No hay forma de 
comprender la experiencia de malestar social o enfermedad si no se participa en algún sentido de ella, si no se planifica y evalúa desde las redes interpersonales del sufrimiento y en el contexto local y etnográfico de acción; es desde esos espacios locales que debe proyectarse la acción más general. Supone el desafío de resignificar el lenguaje y aproximación global, a términos que se vinculen con la historia local y las biografías personales. Los logros narrativos, como una validación de los sufrimientos y una autorización para hablar de ellos, en un despliegue de palabras, gestos y comportamientos, han sido expuestos entre otros por Gorlier (2005) como modos alternativos de encontrar no sólo alivio, sino medios para liberarse. Podemos retomar su hipótesis en torno de los efectos de la conversión religiosa en mujeres que reorganizan sus vidas en torno a un nuevo núcleo identitario a partir de nuevas pertenencias grupales. Healy (2001) por su parte, demuestra que no existe un espacio incontaminado por las relaciones de poder, y que por tanto, el reto de una acción social crítica no consiste tanto en minimizar el poder como en comprender de qué modo puede utilizarse para la liberación y potenciación en los contextos locales de prácticas. El lenguaje y los dispositivos grupales vuelven a ser clave para no ya discutir las relaciones de dependencia, sino el significado de tales relaciones cotidianas de dominación en sus aspectos de malestar y sufrimiento. Al decir de Yeatman (1994, p. 9) "el estado ideal no es la superación de la dominación de una vez por todas, sino unas formas imaginativas y creativas de resistencia positiva a los diversos tipos de dominación". En tanto meta más realista, una política emancipadora consistiría en la creación de las condiciones para el diálogo y la controversia, en un giro reflexivo que distinguimos al comienzo de aquel cambio reflectivo, sin por ello ocultar la existencia de imperativos sociales urgentes.

¿Supone este abordaje un acercamiento a la espiritualidad? Debemos decir que si en la medida que coincidamos con Foucault (2008) en comprender la espiritualidad como una búsqueda, una práctica, una experiencia, a través de la cual el sujeto realiza una transformación por sí mismo. Lo cierto es que la cuestión ha sido tematizada: la espiritualidad debe ser considerada un aspecto de la experiencia vivida, una dimensión de la vida que incluye rituales y creencias, creatividad, acción social y un sentido del tiempo y el espacio. Definida como una actitud frente a la vida, acompaña una búsqueda de significado, propósito y de relaciones moralmente 
plenas con uno mismo, con otros, y la realidad última del universo (FURMAN et al., 2005). Otros han sugerido que la espiritualidad es un proceso de transformación a través del cual los diferentes aspectos de la vida (físico, emocional, laboral, intelectual y racional) son integrados. Envuelve una conexión con uno mismo, otras personas, con la naturaleza, o con un significado mayor, como una deidad o presencia superior. La noción se encuentra fuertemente asociada con la creatividad, el juego, el amor, el perdón, la compasión, la confianza, el respeto, la sabiduría, la fe y el sentido de unidad.

Transformación por uno mismo y a través del 'otro'. Retornemos a la cuestión de la otredad para dar un cierre provisional a este conjunto de interrogantes y reflexiones. El signo de los tiempos parece sugerirnos que la supremacía del yo ha encontrado sus límites, y se debate el aspecto de la condición humana que hace posible por un lado la 'conciencia de sí' y al mismo tiempo nos enfrenta a la vivencia del 'sí mismo' inacabado, incompleto, ante la fractura de la propia autosuficiencia. Al respecto los estudios sobre la temática de la opitulación ${ }^{4}$ constituyen aportes significativos a partir de registros filosóficos y éticos por estos días necesarios. Pues la otredad supone hospitalidad y servicio, ¿porqué? Recogemos un párrafo de Fisher (2011, p.135) para dar cuenta de ello:

...en la proximidad el otro muestra su 'vulnerabilidad'. Y que desde la
invocación del otro muestra su insoslayable 'debilidad', esa que se
manifiesta en la condición del pobre, del enfermo, del discapacitado, del
extranjero, del marginado, que clama y nos reclama en él. Su indigencia
cuestiona toda 'libertad'. De ahí que la posibilidad como deseo ético-
ontológico consiste en restaurar, en la coherencia de los vínculos humanos,
la herida de una relación con el otro.

Las heridas de la desigualdad, del poder y la sumisión no son otra cosa que los objetos de reparación de la política social, bajo el lenguaje que sea: el de la compasión, el de la tarea asistencial, los derechos o la política compensatoria.

\footnotetext{
${ }^{4}$ Procedente del latín opitulatio-onis significa formas de ayuda, hospitalidad, auxilio, asistencia al otro. Cad. de Pesq. Interdisc. em Ci-s. Hum-s., Florianópolis, v.12, n.101, p.31-47 ago/dez 2011
} 


\section{REFERÊNCIAS}

BECK, U.; BECK-GERNSHEIM, E. La individualización. El individualismo institucionalizado y sus consecuencias sociales y políticas. Barcelona: Paidós, 2003.

BERGSON, H. Materia y memoria. Ensayo sobre la relación del cuerpo con el espíritu. Madrid: Librería de Victoriano Suárez y de San Fernando Fe, 1900.

BOURDIEU, P. The Weight of the World: Social Suffering in Contemporary Society. Stanford: Stanford University Press, 1995.

BREILH, J. Epidemiología crítica. Ciencia emancipadora e interculturalidad. Buenos Aires: Lugar Editorial, 2003.

DANANI, C. La gestión de la política social: un intento de aportar a su problematización. En CHIARA, M. y M.M. DI VIRGILIO (Orgs.) La gestión de la política social. Conceptos y herramientas. Buenos Aires: UNGS-Editorial Prometeo, 2009. P. 25-51.

DI LISCIA, M.; BOHOSLAVSKY,E. (Eds.) Instituciones y formas de control social en América Latina 1840-1940. Buenos Aires: Prometeo Libros, 2005.

DOYAL, L.; GOUGH, I. Teoría de las necesidades humanas. Barcelona: Icaria/FUHEM, 1994.

FARMER, P. On suffering and structural violence: a view from below. En: KLEINMAN, A.; DAS, V. y M. LOCK (Eds.). Social Suffering. California: University of California Press, 1997.p. 261-308.

FISHER, R. Entre el hecho religioso y el servicio al hermano. En: ZAMBRANO y DIEZ (Comp.). Prácticas de ayuda. Estudios antropológicos, filosóficos y políticos acerca de la opitulación. Buenos Aires: Miño y Dávila, 2011. p. 133-152.

FOUCAULT, M. La Arqueología del Saber. Buenos Aires: Siglo XXI, 2008.

FROST, L; HOGGETT, P. Human. Agency and social suffering. Critical Social Policy, Londres, vol. 28, n. 4, p. 438-460, nov. 2008.

FURMAN, L.D. et al. A Comparative International Analysis of Religion and Spirituality in Social Work: A Survey of UK and US Social Workers. Social Work Education, Nueva York, vol. 24, n. 8, p. 813-839, 2005.

GORLIER, J. C. Construcción social, identidad, narración. Nuevos enfoques teóricos y el (re) hacer del género. La Plata: Ed. Al Margen, 2005.

HEALY, K. Trabajo social: perspectivas contemporáneas. Madrid: Morata, 2001. 
HELLER, A.; FERENC,F. Biopolítica.Barcelona: Peninsula, 1995.

HIRSCHMAN, A. O.Las pasiones y los intereses. México: Fondo de Cultura Económica, 1978.

KRMPOTIC, C. Medicalización de la vida cotidiana y políticas de salud. En:

KRMPOTIC, C. (Comp.) Cuidados, terapias y creencias en la atención de la salud. Buenos Aires: Espacio Editorial, 2008. p. 17-33.

LUKÁCS, G. Marx, Ontología del ser social. Madrid: Akal, 2007.

MERKLEN, D. Pobres ciudadanos. Las clases populares en la era democrática (Argentina, 1983-2003). Buenos Aires: Gorla, 2005.

SAWAIA, B. O sofrimento ético-político como categoria e análise da dialética exclusão-inclusão. São Paulo: Colóquio Internacional Brasil/França/Portugal: Renda mínima no debate internacional, PUC-SP, 2001.

TOURAINE, A. El regreso del actor. Buenos Aires: EUDEBA, 1987.

YEATMAN, A. Postmodern revisionings of the political. Nueva York: Routledge, 1994.

Artigo:

Recebido em: 15/10/2011

Aceito em: 29/11/2011 